\title{
Efeito do tempo de cura na rigidez de argamassas produzidas com cimento Portland
}

\section{(Effect of the curing time on the stiffness of mortars produced with Portland cement)}

\author{
G. C.R. Garcia, E. M. B. Santos, S. Ribeiro \\ Departamento de Engenharia de Materiais - DEMAR, Escola de Engenharia de Lorena - EEL, Universidade de \\ S. Paulo - USP, Estrada Santa Lucrecia s/n, Bairro Mondezir, C.P. 116, Lorena, SP 12600-970 \\ giselicrg@ppgem.eel.usp.br
}

\begin{abstract}
Resumo
O concreto de cimento Portland é um dos materiais mais usados no mundo inteiro, entretanto, devido a sua estrutura ser muito complexa, torna-se imprescindível estudar suas propriedades com bastante profundidade. O concreto é produzido a partir de uma argamassa, de areia e cimento, com adição de agregados graúdos, sendo que suas propriedades estão basicamente suportadas nessa argamassa de constituição. O objetivo deste trabalho foi estudar a variação da rigidez de duas argamassas de composições com razão cimento:areia de 1:2 e 1:3 em função do tempo de cura, tendo como parâmetro a variação do módulo de Young. Os resultados mostraram que o módulo de Young cresce até atingir o valor máximo no oitavo dia, sendo que nos três primeiros dias esse crescimento é mais acentuado. A análise dos resultados indica que grande parte do processo de hidratação do cimento, com formação das ligações químicas responsáveis pela rigidez da argamassa, acontece nos primeiros dias de cura.

Palavras-chave: Cimento Portland, argamassa, concreto, cura, módulo de Young.
\end{abstract}

\begin{abstract}
Concrete produced with Portland cement is one of building materials most widely used worldwide. However, due to its highly complex structure, its properties require in-depth studies. Concrete is a mortar consisting of a mixture of cement, sand and coarse aggregates, and its properties are represented basically by the mortar base. The aim of this work was to study the change in stiffness of two mortar compositions cured at $25{ }^{\circ} \mathrm{C}$ with a cement-to-sand ratio of 1:2 and 1:3, as a function of curing time using the variation of Young modulus as the measuring parameter. The results showed that Young modulus increases up to a maximum value on the $8^{\text {th }}$ day, and that this increase is more pronounced during the first three days. An analysis of the results indicates that a large part of the cement hydration process, involving the formation of chemical bonds that are responsible for the mortar stiffness, takes place in the early days of curing.
\end{abstract}

Keywords: Portland cement, mortar, concrete, cure, Young modulus.

\section{INTRODUÇÃO}

O concreto é um dos materiais mais usados no mundo todo e pode ser definido como um material macroscopicamente heterogêneo que apresenta propriedades dependentes de suas fases, da relação entre as mesmas, bem como de seus constituintes [1, 2].

A argamassa é constituída de cimento, areia e água, sendo que a areia é considerada um agregado miúdo, logo, o concreto é uma argamassa acrescida de agregados graúdos [1-4]. O tipo de agregado também oferece influência significativa nas propriedades mecânicas do concreto, uma vez que tanto a geometria como a superfície do agregado devem ser considerados [2, 5, 6]. A razão água/cimento talvez seja um dos parâmetros mais relevantes no preparo de argamassas, pois a relação das reações de hidratação com o tempo e as propriedades finais estão intimamente ligadas a essa razão [6-11]. O cimento tipo Portland é o aglomerante mais utilizado na produção de argamassas e concretos. Seus principais constituintes são os silicatos de cálcio: $\mathrm{C}_{3} \mathrm{~S}$ (silicato tricálcico-3CaO. $\mathrm{SiO}_{2}$ ) e $\mathrm{C}_{2} \mathrm{~S}$ ( silicato dicálcico-2CaO. $\mathrm{SiO}_{2}$ ); os aluminatos de cálcio: $\mathrm{C}_{3} \mathrm{~A}$ (aluminato tricálcico-3 $\mathrm{CaO}$. $\mathrm{Al}_{2} \mathrm{O}_{3}$ ) e o $\mathrm{C}_{4} \mathrm{AF}$ (ferroaluminato de cálcio-4CaO. $\mathrm{Al}_{2} \mathrm{O}_{3}$. $\left.\mathrm{Fe}_{2} \mathrm{O}_{3}\right)$, além da gipsita $\left(\mathrm{CaSO}_{4} \cdot 2 \mathrm{H}_{2} \mathrm{O}\right)$ que é adicionada para inibir a tendência à pega instantânea do clínquer devido a reatividade do $\mathrm{C}_{3} \mathrm{~A}$, bem como formar a etringita $(6 \mathrm{CaO}$. $\left.\mathrm{Al}_{2} \mathrm{O}_{3} \cdot \mathrm{SiO}_{2} \cdot 32 \mathrm{H}_{2} \mathrm{O}\right)[12,13]$. A formação de agulhas de etringita começa minutos após a hidratação, e dependendo da proporção alumina-sulfato do cimento, ela pode tornar-se instável e decompor-se formando o monossulfato hidratado, que é mais estável [12-17].

A hidratação dos aluminatos confere o enrijecimento da argamassa, enquanto a dos silicatos, principalmente a do $\mathrm{C}_{3} \mathrm{~A}$, confere o endurecimento ou aumento da resistência, 
principalmente nos estágios iniciais de hidratação $[9,11-$ 13]. Os silicatos de cálcio hidratados são representados genericamente por $\mathrm{C}-\mathrm{S}-\mathrm{H}$ que, juntamente com o hidróxido de cálcio, $\mathrm{Ca}(\mathrm{OH})_{2}$, preenchem o espaço ocupado pela água e pelas partículas de cimento em dissolução [18-20].

O conhecimento das propriedades elásticas de argamassas e concretos, bem como as principais propriedades que caracterizam a rigidez elástica dos materiais, como módulo de Young E, módulo de cisalhamento G, e razão de Poisson $\mu$, são indispensáveis na determinação de várias outras propriedades mecânicas dos materiais [21, 23]. O módulo de elasticidade é uma medida de rigidez que avalia a resistência do material à deformação elástica. Os materiais que deformam muito elasticamente quando sujeitos à solicitações mecânicas, possuem baixo módulo, sendo indicados quando a deformação é apenas transitória. No entanto, na maioria das aplicações não se deseja a ocorrência de deflexões, ou seja, os materiais devem apresentar alto módulo de elasticidade [22]. Geralmente os métodos utilizados para tal medida podem ser divididos em duas classes: métodos estáticos e métodos dinâmicos. Os métodos estáticos consistem em solicitar mecanicamente um corpo por meio de aplicação de tensão, podendo ser de tração, compressão, flexão ou torção, com baixas velocidades de aplicação de carga [21, 22]. Os métodos dinâmicos consistem basicamente na determinação da freqüência natural de um corpo de prova pelo emprego da energia das ondas elásticas e na medição da velocidade de propagação de onda através de um corpo. As tensões aplicadas nesse método são muito menores que a no primeiro, garantindo que o material está no regime elástico [22]. Dentre os métodos dinâmicos, destaca-se a técnica de ressonância mecânica de barras [24-27]. O princípio do método da ressonância mecânica de barras consiste no estímulo de um corpo de prova com frequiência variável, na busca das freqüências de ressonância de vibração desse corpo e por meio de relações matemáticas determinam-se os módulos elásticos. Essas relações são específicas para cada geometria de corpo de prova [26-28]. Ida e Foster são referenciados como proponentes da metodologia referida [24].

O objetivo desse trabalho foi avaliar a evolução do módulo de Young com o tempo de cura de argamassas de duas composições diferentes utilizando o método não destrutivo de ressonância de barras a fim de se obter uma análise da resistência à deformação elástica desses materiais.

\section{MATERIAIS E MÉTODOS}

Para obtenção das amostras foram utilizados areia média lavada, cimento Portland CPII-E-32 da Companhia de Cimento Montes Claros, e água potável. Foram preparadas duas composições mássicas em termos de cimento, uma $1: 2$ e outra $1: 3$. A quantidade de água calculada foi baseada na razão água:cimento de 0,46 para $1: 2$, e 0,56 para $1: 3$, a fim de que as duas argamassas apresentassem o mesmo comportamento reológico [9-11]. A areia e o cimento foram pesados previamente e colocados em uma argamassadeira planetária de laboratório com capacidade nominal de $5 \mathrm{~L}$, na qual foram misturados por $3 \mathrm{~min}$ a $60 \mathrm{rpm}$. Em seguida a água foi adicionada lentamente, com a argamassadeira trabalhando, ficando mais 1 min na mesma rotação, e depois $1 \mathrm{~min}$ a $120 \mathrm{rpm}$. Enquanto a mistura foi vertida em um molde de aço inoxidável, o mesmo foi vibrado em uma mesa vibratória, sendo depois curado em uma câmara com atmosfera saturada de água a $25{ }^{\circ} \mathrm{C}$ por 24 h. Em cada moldagem foram obtidas 5 barras prismáticas com dimensões $25 \times 25$ x $150 \mathrm{~mm}^{3}$. Após $24 \mathrm{~h}$ as amostras foram desmoldadas e pesadas para a realização da primeira medida de módulo elástico. Imediatamente após a realização das medidas, as amostras voltaram para cura, sendo esse procedimento de pesagem e medida de módulo de Young repetido até completos 28 dias de cura, tendo sido tomado o devido cuidado para minimização de erros experimentais. Para medidas de módulo de Young foi utilizado um equipamento automático, baseado no método da ressonância de barras. Essa técnica consiste na passagem de uma vibração mecânica pela amostra através de um transdutor piezelétrico. A vibração é sentida no outro transdutor e transformada em sinal elétrico, transmitida para o programa computacional que registra os picos de ressonância, e de modo interativo calcula o módulo elástico e de cisalhamento, assim como a razão de Poisson [26-28]. O sistema utilizado foi da ATCP/ ME-C1198-91 obedecendo à norma ASTM C1198-91 e a faixa de frequiência adotada na varredura das medidas foi de 1 a $30 \mathrm{kHz}$.

Para análise das fases nas amostras curadas por 28 dias, foi utilizado um difratometro de raios X Siemens, radiação $\mathrm{Cu}-\mathrm{k} \alpha$, com intervalo angular de 5 a $75^{\circ}$ e passo de $0,05^{\circ}$. Para análise de microestrutura, as amostras foram recobertas com um filme fino de carbono e observadas em microscópio eletrônico de varredura LEO 1450 VP, no modo elétrons secundários.

\section{RESULTADOS E DISCUSSÃO}

A Tabela I mostra os resultados de módulo de Young nos dias em que as medidas apresentaram resultados mais significativos. Para cada composição foram moldadas 5 amostras; sendo assim, os resultados apresentados são médias com seus respectivos desvios.

Do primeiro para o segundo dia o crescimento do módulo de Young foi de $37,05 \%$ para a argamassa de composição $1: 2$, e de $67,37 \%$ para $1: 3$, mostrando que o crescimento da última é mais acentuado. A Fig. 1 mostra os resultados médios das medidas de módulo de Young, (a), e perda de massa, (b), das amostras de argamassas de composições 1:2 e 1:3 em função do tempo de cura.

Tanto o módulo de Young quanto a perda de massa mostram uma mesma tendência: aumentam mais acentuadamente nos primeiros dias de cura, o crescimento passa a ser gradativo, chegando à estabilização após o oitavo dia para o módulo de Young. Já para a perda de massa nota-se que mesmo para tempos mais longos, apesar de sua taxa de crescimento 
Tabela I - Medidas do módulo de Young das argamassas 1:2 e 1:3. [Table I-Measurements of Young modulus of mortars 1:2 and 1:3.]

\begin{tabular}{ccccccc}
\hline & \multicolumn{6}{c}{ Módulo de Young E (GPa) em função do tempo de cura } \\
Argamassa & \multicolumn{7}{c}{ (dias) } \\
\cline { 2 - 7 } & 1 & 2 & 3 & 7 & 8 & 28 \\
\hline \multirow{2}{*}{$1: 2$} & 17,38 & 23,82 & 25,05 & 27,17 & 27,51 & 28,26 \\
& $\pm 0,28$ & $\pm 0,45$ & $\pm 0,15$ & $\pm 0,32$ & $\pm 0,30$ & $\pm 0,38$ \\
& 10,48 & 17,54 & 18,39 & 20,41 & 20,61 & 21,16 \\
$1: 3$ & $\pm 0,52$ & $\pm 0,70$ & $\pm 0,89$ & $\pm 1,01$ & $\pm 1,17$ & $\pm 1,24$ \\
\hline
\end{tabular}
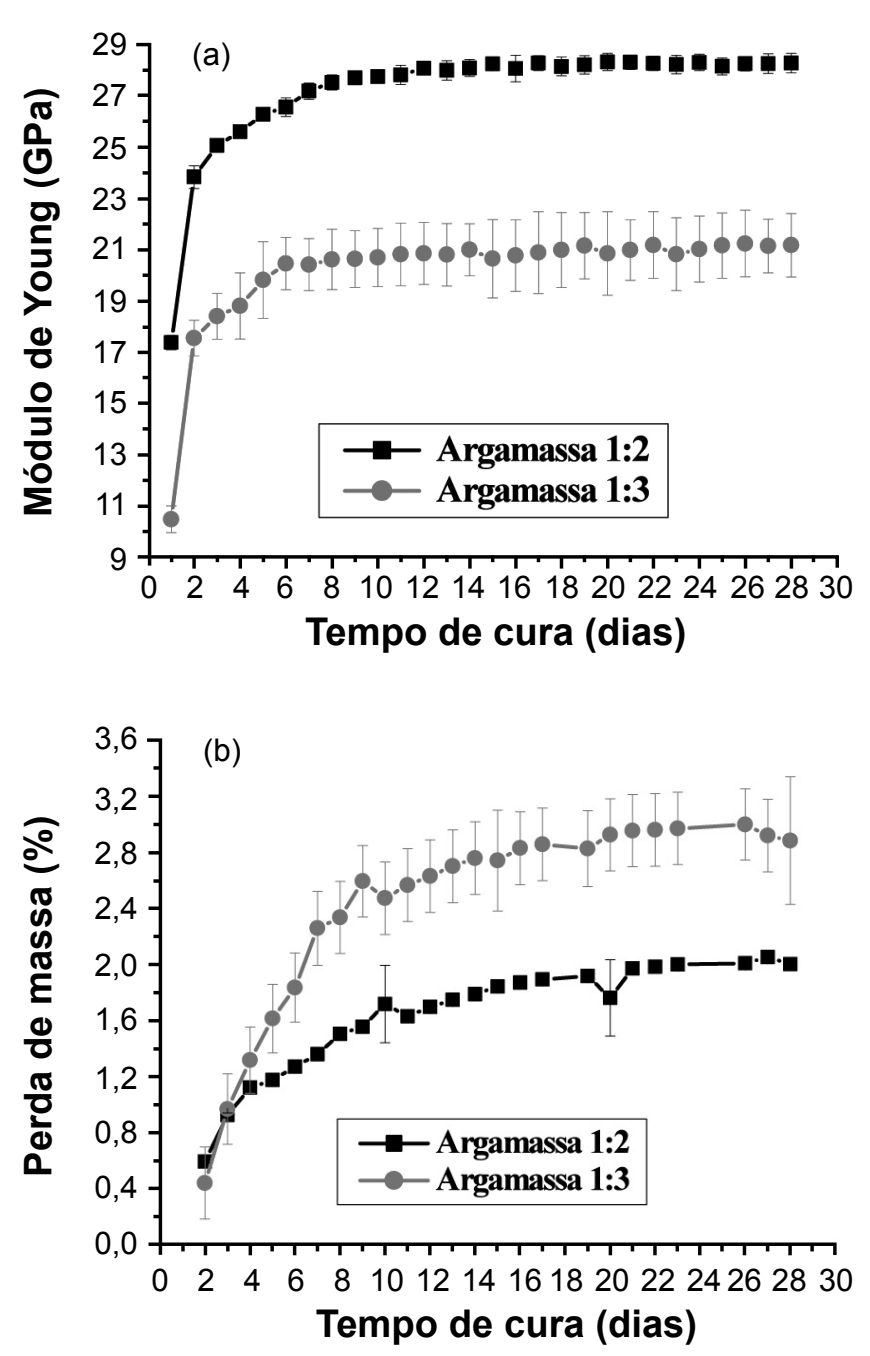

Figura 1: Módulo de Young (a) e perda de massa (b) em função do tempo de cura das argamassas 1:2 e 1:3.

[Figure 1: Young modulus (a) and weight loss (b) as a function of curing time of the mortars 1:2 and 1:3.]

diminuir, ela continua variando durante todo o período de avaliação. $\mathrm{O}$ aumento do módulo elástico é promovido pela formação das ligações dos hidratos produzidos pelas reações entre o cimento e a água adicionada para a produção das argamassas tornando-as cada vez mais rígida, principalmente até o oitavo dia de cura. Após o oitavo dia pode-se concluir que praticamente todas as reações se completaram e as argamassas estão com sua estrutura consolidadas. Como era de se esperar a argamassa com maior quantidade de cimento apresentou maiores valores de módulos de Young. Isso é mostrado como sendo efeito das quantidades de ligações de hidratação na estrutura da argamassa [8, 17-19]. A perda de massa observada na Fig. $1 \mathrm{~b}$ pode ser atribuída à perda de água por evaporação. Durante a cura, as amostras ficavam em uma câmara hermeticamente fechada com atmosfera saturada de água e, quando eram retiradas desta câmara para realização das medidas, a pressão parcial da água no ambiente era inferior à da câmara, causando perda de massa. A argamassa de composição 1:3 perdeu mais água que a 1:2, devido, obviamente, à sua composição inicial com maior quantidade de água para moldagem: 0,56 e 0,46 respectivamente. Ressalta-se que para a argamassa 1:3 foi necessário usar uma quantidade maior de água que a 1:2, a fim de obter a mesma consistência para a moldagem das duas composições [9-11].

A Tabela II mostra os resultados de porosidade aparente, absorção de água e massa específica aparente, determinados segundo a NBR 6220. A menor porosidade e conseqüentemente menor absorção de água resulta em maior resistência para a argamassa 1:2.

A argamassa 1:2 é mais rígida que a 1:3 por dois motivos muito importantes: o primeiro é a maior quantidade de cimento usada, que traduz em mais ligações de hidratação resultando em maior rigidez; o segundo é que pelo fato

Tabela II - Porosidade aparente, absorção de água e massa específica aparente das argamassas 1:2 e 1:3.

[Table II - Apparent porosity, water absorption and density of mortars $1: 2$ and $1: 3$.]

\begin{tabular}{cccc}
\hline Argamassa & $\begin{array}{c}\text { Porosidade } \\
\text { Aparente } \\
(\%)\end{array}$ & $\begin{array}{c}\text { Absorção de } \\
\text { Água (\%) }\end{array}$ & $\begin{array}{c}\text { Massa } \\
\text { Específica } \\
\text { Aparente } \\
\left(\mathrm{g} / \mathrm{cm}^{3}\right)\end{array}$ \\
\hline $1: 2$ & $12,31 \pm 0,92$ & $5,75 \pm 0,43$ & $2,14 \pm 0,002$ \\
$1: 3$ & $19,43 \pm 0,33$ & $9,66 \pm 0,20$ & $2,01 \pm 0,008$ \\
\hline
\end{tabular}


de ser produzida com menor quantidade de água é menos porosa, conforme pode ser visto na Tabela II. Também é importante ressaltar que o módulo de elasticidade é função das ligações químicas envolvidas nos materiais e de sua porosidade [6-8, 12].

A Fig. 2 mostra os difratogramas de raios $\mathrm{X}$ das argamassas 1:2 (a) e 1:3, (b) obtidos a partir do pó das amostras após 28 dias completos de cura. Nas duas composições foram identificadas as mesmas fases diferindo apenas na porcentagem de intensidade dos picos, que se deve às diferentes proporções das matérias-primas. A fase caracterizada pela etringita está presente nas duas composições em pequena proporção, sendo que na $1: 3$ seus picos são pouco mais intensos que na 1:2. Os picos indexados referentes ao quartzo identificam a areia presente nas duas composições. Picos de silicatos, aluminatos e de hidróxido de cálcio hidratados também foram encontrados.

A Fig. 3 mostra as micrografias obtidas por microscopia eletrônica de varredura no modo elétrons secundários das argamassas nas composições 1:2 (a), (c) e (e), e 1:3 (b) e (d), da superfície de fratura das amostras com tempo de cura superior a 28 dias. Em (a) e (b) observa-se a matriz formada pelos silicatos hidratados. Em (c) e (d) nota-se a presença de etringita, pois a mesma exibe microestrutura na forma de agulhas [14-16]. Essas agulhas puderam ser melhor observadas em ampliações da ordem de 5000 vezes para 1:3, (d), e de 10000 vezes para 1:2, (e).

Por meio de uma análise comparativa das micrografias com os difratogramas de raios $\mathrm{X}$, foi possível observar que as agulhas de etringita são mais nítidas na argamassa 1:3 que 1:2. Este fato deve estar correlacionado com a maior proporção de água na 1:3 ocasionando, num primeiro momento, uma maior taxa de hidratação que leva à formação da fase etringita, e que decorrido os 28 dias ela não se decompôs totalmente. Como a resistência mecânica em argamassas é conferida pelas reações de hidratação dos silicatos [6-13,17-20], e na argamassa $1: 2$ a quantidade relativa de cimento foi maior, a proporção desses silicatos hidratados também deve ter sido maior, uma vez que o módulo de Young também foi maior, o que proporciona maior resistência à deformação elástica.

\section{CONCLUSÕES}

A quantidade relativa de cimento, bem como a de água, interfere diretamente no módulo de Young das argamassas. A argamassa de composição 1:2 apresentou menor porosidade, menor perda de massa e módulo de Young superior, em torno de $30 \%$, à argamassa 1:3, evidenciando que a 1:2 é mais resistente à deformação elástica, além de seus resultados serem mais confiáveis com relação aos desvios obtidos. Os resultados encontrados apontam que no oitavo dia as amostras já atingiram seu estado de máxima resistência à deformação elástica. $\mathrm{O}$ fato de a amostra ser retirada da cura, onde a atmosfera era saturada de água, para realização das medidas, também contribuiu para perda de massa das mesmas. A técnica da ressonância mecânica de barras utilizada para medidas de módulo de Young é bastante confiável quanto à reprodutibilidade e precisão dos valores medidos, além de ser um método não destrutivo e rápido que aumenta as possibilidades de caracterização de materiais como argamassas e concretos.

\section{AGRADECIMENTOS}

À FAPESP (Proc. 07/55964-3), ao CNPq pela bolsa de Mestrado (Proc. 135921/2008-2), pela bolsa de Doutorado (Proc. 140202/2009-9), e pela bolsa de Produtividade em Pesquisa (Proc. 302387/2007-2). Ao Centro Técnico Aeroespacial, CTA, pelas análises de MEV.

\section{REFERÊNCIAS}

[1] P. Stroeven, Cement \& Concrete Composites 22 (2000) 331-341.

[2] T. Sadowski, G. Golewski, Computacional Mater. Sci. 43 (2008) 119-126.

[3] S. Ribeiro, J. A. Rodrigues, $52^{\circ}$ Congresso Brasileiro de Cerâmica, Florianópolis, SC (2008) 1-12.

[4] Z. Sun, E. J. Garboczi, S. P. Shah, Cement Concrete Composites 29 (2007) 22-38.

[5] A. R. Mohamed, W. Hansen, Cement Concrete Composites 21 (1999) 349-359.

[6] S. Erdogdu, S. Kurbetci, Cement \& Concrete Composites 27 (2005) 818-822.

[7] A. Boumiz, C. Vernet, F. C. Tenoudji, Adv. Cem. Bas. Mat. 3 (1996) 94-106.

[8] J. M. Chi, R. Huang, C. C. Yang, J. J. Chang, Cement Concrete Composites 25 (2003) 197-205.

[9] S. Ribeiro, J. A. Rodrigues, Cerâmica 55 (2009) 181189.

[10] S. Ribeiro, E. M. B. Santos, G. C. R. Garcia, J. A. Rodrigues, Proc. $5^{\text {th }}$ Int. Mater. Symp. (MATERIAIS 2009) Lisboa, Portugal 1 (2009) 1-6.

[11] J. C. Nadeau, Cement Concrete Res. 32 (2002) 481490.

[12] P. K. Mehta, P. J. M. Monteiro, Concreto: estrutura, propriedades e materiais, PINI, S. Paulo, SP (1994) 573.

[13] P. J. M. Monteiro, "Microstructure of concrete and its influence on the mechanical properties", Tese Dr., University of California, Berkeley, CAL, EUA (1985) 153.

[14] J. Chen, M. Jiang, Construction and Building Mater. 23 (2009) 812-816.

[15] H. F. W. Taylor, C. Famy, K. L. Scrivener, Cement Concrete Res. 31 (2001) 683-693.

[16] M. Thomas, K. Folliard, T. Drimalas, T. Ramlochan, Cement and Concrete Res. 38 (2008) 841-847.

[17] F. A. E. Freitas, "Microfissuração e evolução da hidratação de concreto do cimento Portland, com e sem adição de escória, por meio de análise de imagens", Diss. Mestrado, Universidade de Campinas, Campinas, SP (2001) 185. 

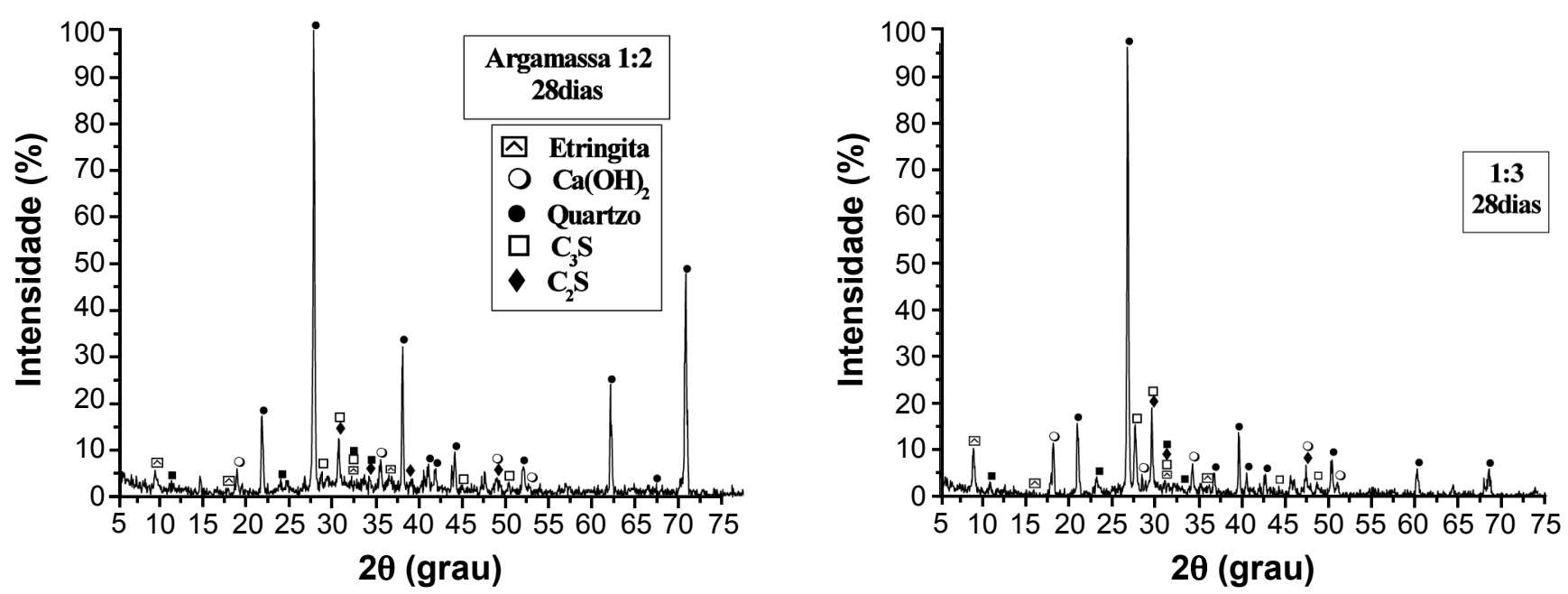

Figura 2: Difratogramas de raios X das argamassas de composição 1:2 e 1:3, com 28 dias de cura.

[Figure 2: X-ray diffraction patterns of the mortar composition 1:2 and 1:3, with 28 days of age.]

(a)

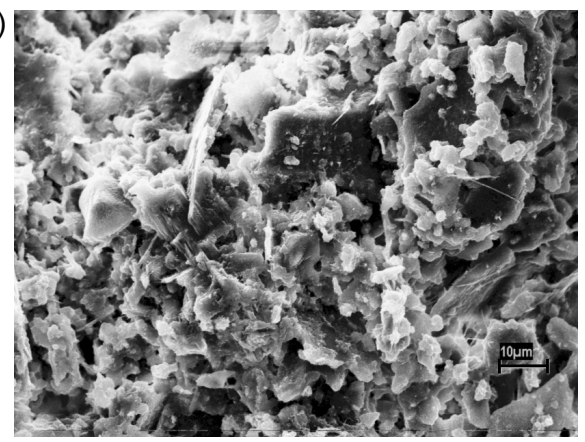

(c)

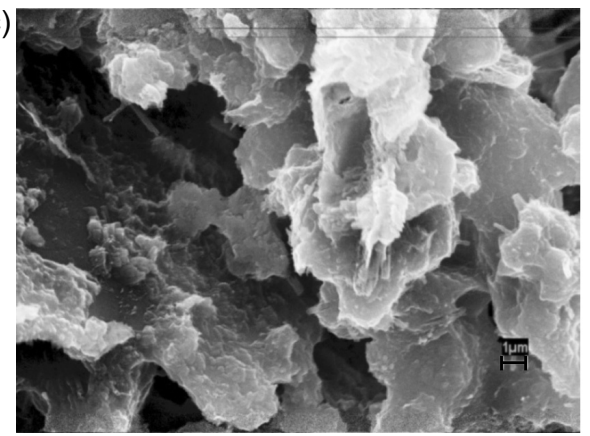

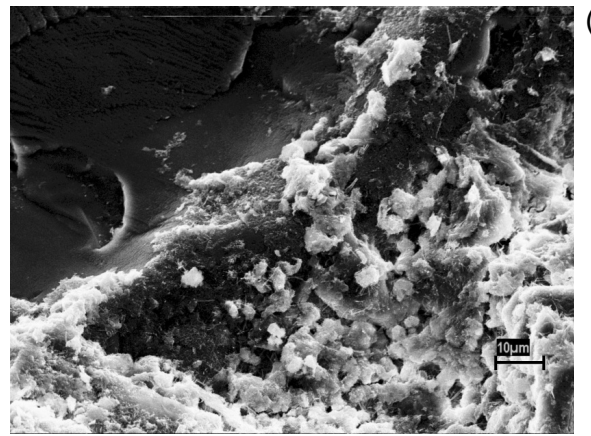

(b)

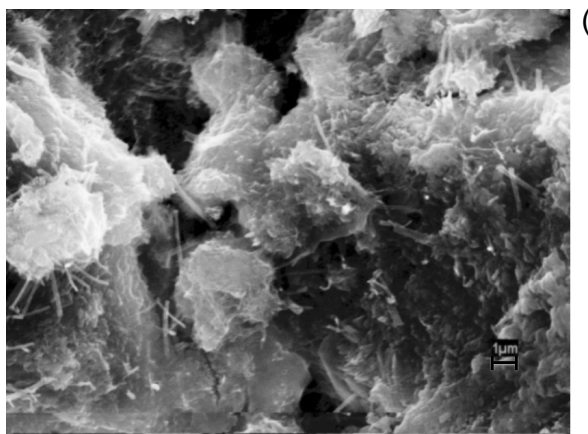

$(d)$

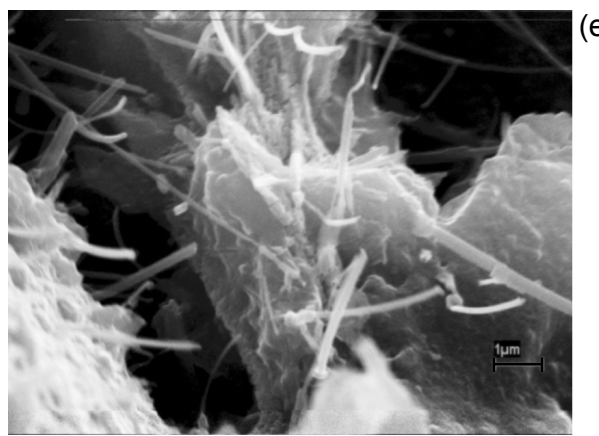

(e)

Figura 3: Micrografias, obtidas por microscopia eletrônica de varredura no modo elétrons secundários, da superfície de fratura das argamassas nas composições 1:2, (a), (c) e (e), e 1:3, (b) e (d).

[Figure 3: Micrographs obtained by scanning electron microscopy in secondary electron mode of the fracture surface of mortar in the compositions 1:2, (a), (c) and (e), and 1:3, $(b)$ and $(d)$.] 
[18] O. Bernard, F. J. Ulm, E. Lemarchand, Cement Concrete Res. 32 (2003) 1293-1309.

[19] D. P. Bentz, Cement Concrete Res. 38 (2008) 196-204. [20] C. Famy, A. R. Brough, H. F. W. Taylor, Cement Concrete Res. 33 (2003) 1389-1398.

[21] W. Hayden, W. G. Moffatt, J. Wulff, Mechanical Behavior, John Wiley \& Sons, New York, EUA (1965).

[22] E. D. Zanotto, A. R. Migliory Jr., Cerâmica 37, 247 (1991) 7-16.

[23] M. T. P. Aguilar, A. P. Silva, E. C. S. Corrêa, P. R. Cetlin, Anais XVII CBECIMAT, Foz do Iguaçu, PR (2006) 6780-679.

[24] E. Schreiber, A. L. Orson, N. Soga, "Dynamic Ressonance Method for measuring the elastic moduli of solids", McGraw-Hill (1973) 82-125.
[25] D. N. Boccaccini, M. Romagnoli, E. Kamseu, P. Veronesi, C. Leonelli, G. C. Pellacani, J. Eur. Ceram. Soc. 27 (2007) 1859.

[26] ASTM: E 1875-00, "Standard Test Method for Dynamic Young's Modulus, Shear Modulus,and Poisson's Ratio by Sonic Resonance" (2002).

[27] ASTM: C 1198-91, "Standard Test Method for Dynamic Young's Modulus, Shear Modulus, and Poisson's Ratio for advanced ceramics by Sonic Resonance" (2002).

[28] A. E. M. Paiva, J. A. Rodrigues, Anais $45^{\circ}$ Congresso Brasileiro de Cerâmica, Florianópolis, SC (2001) 301-313.

[29] M. Ghrici, S. Kenai, M. Said-Mansour, Cement Concrete Composites 29 (2007) 542-549.

[30] J. Kim, Y. Lee, S. Yi, Cement Concrete Res. 34 (2004) 507-519.

(Rec. 11/11/2009, Ac. 22/05/2010) 